\title{
The Migration of Luteinizing Hormone-Releasing Hormone Neurons in the Developing Rat Is Associated with a Transient, Caudal Projection of the Vomeronasal Nerve
}

\author{
Keiko Yoshida, ${ }^{1}$ Stuart A. Tobet,, ${ }^{1,2}$ James E. Crandall, ${ }^{1}$ Tanya P. Jimenez, ${ }^{1}$ and Gerald A. Schwarting ${ }^{1,2}$ \\ 'The Shriver Center, Waltham, Massachusetts 02154 and 2 Program in Neuroscience, Harvard Medical School, \\ Boston, Massachusetts 02115
}

\begin{abstract}
Luteinizing hormone-releasing hormone (LHRH) neurons originate in the olfactory placode and vomeronasal organ and migrate to the brain from embryonic day 14 (E14) in the rat. We investigated the development of the vomeronasal nerve and its role as a guide for migrating LHRH neurons. Using fluorescent, lipophilic dye tracing methods, we observed axons that emerge from the vomeronasal organ and cross the nasal septum as several large fascicles. At E14-15, these fascicles converge as they enter the region of the cribriform plate and subsequently disperse, projecting dorsally and caudally across the olfactory bulb and rostral forebrain. At E16, the dorsal branch of the vomeronasal nerve forms a more tightly fasciculated projection; the caudal fibers remain dispersed, extending along the medial forebrain. The number of caudally directed axons decreases during development, leaving four or five present at postnatal day 4 (P4). Immunohistochemical studies indicate that the vomeronasal nerve can be divided into four spatialiy distinct subpopulations of fibers. One subset, composed of caudal fibers that terminate in the lamina terminalis, selectively expresses TAG- $\dot{1}$, a transient axonal surface glycoprotein and PSA-N-CAM, a highly polysialated form of neural cell adhesion molecule. The extension and subsequent retraction of this branch of the vomeronasal nerve corresponds spatially and temporally with the migration of LHRH neurons from the nasal cavity to the brain. Our studies show that between E14 and E18, LHRH neurons migrate in contact with the TAG-1+, PSA-N-CAM ${ }^{+}$caudal branch of the vomeronasal nerve.
\end{abstract}

[Key words: vomeronasal, luteinizing hormone-releasing hormone (LHRH), migration, axon guidance, TAG-1, PSAN-CAM]

The migration of neuronal precursors to their final locations and the projection of axons to their appropriate targets are two critical events in neural development that require cell-cell and cellmatrix interactions. In both the cerebral cortex and the cerebellum, cells have been shown to utilize radial glial processes as

Received May 4, 1995; revised July 18, 1995; accepted Aug. 4, 1995.

We thank M. Yamamoto for monoclonal antibody 4D7, R. Benoit for the anti-LHRH antibody LR1, and E. Raabe, for helpful discussion and critical reading of the manuscript. This work was supported by National Institutes of Health Grant DC 00953 and HD 05515

Correspondence should be addressed to Gerald A. Schwarting. The Shriver Center, 200 Trapelo Road, Waltham, MA 02154.

Copyright $\subset 1995$ Society for Neuroscience $0270-6474 / 95 / 157769-09 \$ 05.00 / 0$ guides in migration (Rakic, 1990; Hatten, 1993). However, in the neural crest, precursor cells must use a series of cellular and extracellular matrix cues to reach their destinations (BronnerFraser, 1992). Two patterns of initial axon outgrowth have been described. For example, chick motoneurons project axons from the spinal cord to the limb, where they intermingle, then segregate into new bundles that extend to correct targets (Landmesser et al., 1990). Another common mechanism for establishing correct connections during development is exuberant outgrowth followed by pruning of axons projecting to inappropriate targets (Cowan et al., 1984). In the E15 rat, transient olfactory axons extend through the telencephalon into the ventricular zone. (Santacana et al., 1992; Gong and Shipley, 1995).

The vomeronasal organs (VNOs) are chemosensory structures that are located bilaterally, ventral to the nasal septum in the rat and are connected via the vomeronasal nerves (VNNs) to the accessory olfactory bulbs (AOBs) (Halpern, 1987; Garrosa, 1992). As part of the accessory olfactory system, the VNO is involved in a variety of endocrinologically related functions including pheromonal responses, mating, maternal behavior and maternal recognition (Meredith, 1986; Wysocki and Meredith, 1986; Halpern, 1987). Although, by many criteria, the accessory olfactory system is not functionally mature until the second or third postnatal week, the embryonic pattern of axon outgrowth is of considerable interest because of its possible association with the migration of luteinizing hormone-releasing hormone (LHRH) containing neurons.

Vomeronasal nerves have been included as part of a complex of olfactory fibers that participates in LHRH cell migration (Wirsig and Getchell, 1986; Schwanzel-Fukuda and Pfaff, 1989; Wray et al., 1989; Murakami et al., 1991; Schwanzel-Fukuda et al., 1992; Daikoku et al., 1993). LHRH neurons originate in the olfactory placode (Norgren and Lehman, 1991; Akutsu et al., 1992; Norgren and Gao, 1994) and migrate to their targets in the brain, although the exact mechanisms involved remain unclear. The fibers associated with LHRH neuron migration have been demonstrated to express the neural cell adhesion molecule, NCAM, (Schwanzel-Fukuda et al., 1992, 1994; Norgren and Brackenbury, 1993), a highly polysialated form of neural cell adhesion molecule, PSA-N-CAM, (Murakami et al., 1991), peripherin (Wray et al, 1994), and the CC2-immunoreactive olfactory glycoconjugate (Tobet et al., 1992). We report here that early in development, a transient population of VNN fibers turns caudally from its dorsal trajectory and extends along the ventromedial aspect of the OB into the rostral forebrain. LHRH neuron 

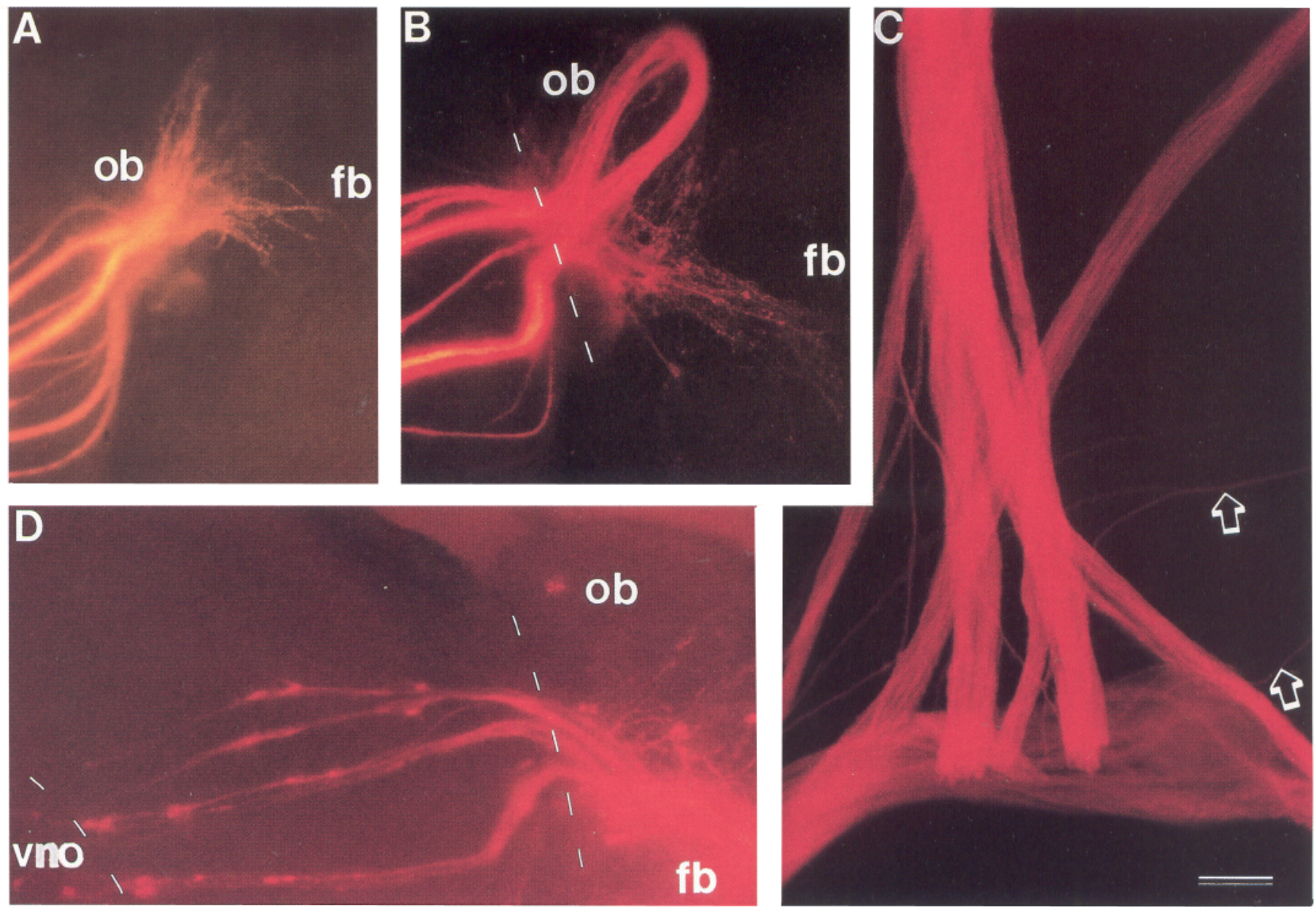

Figure 1. DiI labeling of the developing vomeronasal projection. Vomeronasal nerves emerge from the VNO at day $15(A)$ as distinct fascicles that merge at the cribriform plate (dotted line). Dispersed VNO fibers project dorsally across the olfactory bulb $(o b)$ and caudally into the forebrain $(f b)$. At E16 $(B)$, many new fibers have been added to the dorsal branch, which has begun to fasciculate (in this case into two distinct bundles). Few fibers have been added to the caudal branch which has grown in length but remains defasciculated. At P4 $(C)$, the dorsal branch of the VNN is tightly fasciculated, but the caudal branch consists of only a few isolated fibers (arrows). DiI placed in the medial forebrain at E16 (D), labels axon bundles emerging from the VNO (dotted line at left), along the nasal septum, crossing the cribriform plate (dotted line at right) and along the medial surface of the olfactory bulb and forebrain. Cells within the vomeronasal nerves are also labeled. Rostral is to the right. Scale bar in $C$, $100 \mu \mathrm{m}$; same scale bar represents $200 \mu \mathrm{m}$ in $A, B$, and $D$.

migration across the medial olfactory bulb and forebrain is associated with this caudal branch of the VNN.

\section{Materials and Methods}

DiI labeling of vomeronasal nerves. Pregnant Sprague-Dawley rats at 14,15 , and $16 \mathrm{~d}$ of gestation and $4 \mathrm{~d}$ old pups (Zivic-Miller Laboratories) were anesthetized with a 1:1 mixture of ketamine:xylazine at a dose of $50 \mathrm{mg} / \mathrm{kg}$ ketamine with $10 \mathrm{mg} / \mathrm{kg}$ xylazine. Embryos and pups were perfused first with $10 \mathrm{ml}$ of cold phosphate-buffered saline $(0.05$ $\mathrm{M} \mathrm{pH} \mathrm{7.4),} \mathrm{followed} \mathrm{by} 20 \mathrm{ml}$ of cold $2 \%$ paraformaldehyde in $0.1 \mathrm{M}$ phosphate buffer ( $\mathrm{pH} \mathrm{7.4)}$ at a rate of $1-4 \mathrm{ml}$ per minute. The embryos and pups were then decapitated and postfixed in paraformaldehyde overnight.

1,1'-Dioctadecyl-3,3,3',3'-tetramethylindocarbocyanine perchlorate (DiI, Molecular Probes), a lipophilic fluorescent dye, was used to label vomeronasal nerve fibers. For VNN labeling, the lower jaws were removed and the VNOs were exposed ventrally by dissecting through the soft palate. A crystal of DiI on the tip of a pulled Pasteur pipette was placed into each VNO. For the rostral forebrain injections, a coronal cut was made just anterior to the preoptic area. Crystals of DiI were placed along the medial walls of the septal region. The heads were then put into 6-well cell culture dishes filled with $0.05 \%$ sodium azide in PBS and kept at $37^{\circ} \mathrm{C}$ for $4-14 \mathrm{~d}$. For analysis, each head was cut along the mid-sagittal plane, placed medial side down on a petri dish and analyzed on an inverted fluorescent microscope (Zeiss IM35) equipped with rhodamine filters.

Antibodies. CC1 and CC6 antibodies have been previously described (Schwarting et al., 1992, 1994). P-Path, a monoclonal IgM class antibody, has been shown to recognize a set of 9-0 acetyl gangliosides (Yamamoto et al., 1994). LR1, an anti-LHRH antibody, was a generous gift from Dr. Robert Benoit. 5A5 is a monoclonal IgM antibody against polysialated forms of NCAM (PSA-N-CAM) and was obtained from the Developmental Studies Hybridoma Bank maintained by the Department of Pharmacology and Molecular Sciences, The Johns Hopkins University School of Medicine, Baltimore, MD 21205 and the Department of Biology, University of Iowa, Iowa City, IA 52242 under contract NO1-HD-2-3144 from the NICHD. 4D7 is a monoclonal antibody reacting with the SNAP, TAG-1 adhesion molecule (Yamamoto et al., 1986).

Whole mount immunostaining. Four day old pups and pregnant Sprague-Dawley rats at 16 and $18 \mathrm{~d}$ of gestation were perfused and fixed as described above. Olfactory bulbs and rostral forebrain were carefully dissected out and further cut along the midline into right and left halves. Whole mount immunostaining was performed at $4^{\circ} \mathrm{C}$, using a modified technique from Wirsig-Wiechmann (1993). Briefly, the tissue was placed in $1 \%$ sodium borohydride in PBS for $2-4 \mathrm{hr}$, followed by an $18 \mathrm{hr}$ rinse in several changes of PBS. The tissue was then immersed in a preincubation solution of $0.3 \%$ Triton $\mathrm{X}-100 / 1 \%$ normal goat serum/PBS for $2 \mathrm{hr}$. The brain halves were sequentially incubated in pri- 
mary antibody for $72 \mathrm{hr}$, rinsed in a few changes of PBS for $6 \mathrm{hr}$, treated with secondary antibody for $16 \mathrm{hr}$, rinsed in PBS for $2 \mathrm{hr}$, and then developed with 3-amino-9-ethylcarbazole (AEC, Sigma) chromogen and/or Vector blue (Vector Laboratories) solution for $20 \mathrm{~min}$. P-Path, TAG-1, 5A5, CC6, and CC1 antibodies were used with peroxide-conjugated goat anti-mouse IgM (Boehringer Mannheim) and AEC chromogen solution $(0.0003 \%$ hydrogen peroxide $/ 5.55 \%$ AEC solution of $0.04 \%$ AEC in $N, N$-dimethylformamide in $0.01 \mathrm{M}$ acetate buffer $\mathrm{pH}$ 5.2), LHRH neurons were visualized by incubating tissue with LR1 antibody, alkaline phosphatase-conjugated goat anti-rabbit IgG (Boehringer Mannheim), and Vector Blue alkaline-phosphatase substrate solution (prepared according to directions). Whole mount double-labeling was performed by combining the appropriate antibodies and staining first with AEC chromogen solution, rinsing with PBS and then placing the tissue into the Vector blue solution. All tissues were kept in PBS at $4^{\circ} \mathrm{C}$ after staining.

Slide immunohistochemistry. Embryonic day 16 rat tissue was obtained and fixed as described above. After postfixation, the heads were placed in $25 \%$ sucrose in $0.05 \mathrm{M}$ PBS at $4^{\circ} \mathrm{C}$ for $24 \mathrm{hr}$. The skin, lower jaw, and tongue were removed and serial, horizontal sections $(20 \mu \mathrm{m}$ thick) were cut in a cryostat and thaw mounted on gelatin-coated slides. Immunohistochemistry was performed as described in Yamamoto et al. (1985). Briefly, sections were thawed and rehydrated with 1\% NGS/ PBS for $10 \mathrm{~min}$. The tissue was then exposed to TAG-1 antibody diluted in $1 \%$ NGS/PBS overnight at $4^{\circ} \mathrm{C}$. After three 10 min washes with $1 \%$ NGS/PBS, sections were incubated with fluoroscein-conjugated goat anti-mouse IgM (Boehringer-Mannheim) in 1\% NGS in PBS at room temperature for $2 \mathrm{hr}$. Sections were washed with three $10 \mathrm{~min}$ rinses of PBS, dipped in distilled water and coverslipped with a 1:1 mixture of glycerol and a solution of $4 \mathrm{mg} / \mathrm{ml}$ paraphenylenediamine in $0.2 \mathrm{M}$ carbonate buffer ( $\mathrm{pH}$ 9.0). Double-labeling for TAG-1/LHRH was performed by using TAG-1 antibody and LR1 antibody followed by fluorescein-conjugated goat anti-mouse IgM and Cy3-conjugated goat antirabbit IgG (Jackson Immunochemicals) antibodies.

\section{Results}

\section{Embryonic development of the VNN}

The development of the VNO axon projections was investigated by placing DiI crystals in the rat VNO on days E14 through P4. At all ages examined, the vomeronasal nerve emerged from the VNO and traversed the nasal septum as six to eight large axon bundles that converge before crossing the cribriform plate. At E14 and E15, after the VNN fascicles merged at the cribriform plate, they defasciculated, extending dorsally across the olfactory bulb and caudally into the telencephalon. At E15, the caudal projection was remarkably similar in number and length of fibers to the dorsal projection (Fig. 1A). At E16, the number of fibers projecting dorsally increased dramatically and most of these fibers refasciculated into one or two bundles that continued along the medial surface of the olfactory bulb to the AOB (Fig. 1B). At this age, the caudal projection increased only moderately in fiber numbers but expanded considerably into the forebrain (Fig. $1 B)$. Most of these caudal fibers remained defasciculated as they crossed the rostral forebrain, extending along the medial septal wall to the lamina terminalis.

Placement of DiI crystals in the VNO at P4 demonstrated that the main VNN projecting dorsally was tightly fasciculated, although a few single fibers were visible along the main nerve bundle (Fig. 1C). In contrast to the numerous caudally directed VNN fibers that were visible at E16, only four or five remained at P4.

DiI injections in the forebrain of E16 rats were performed to confirm the vomeronasal origin of these caudally directed fibers. Four days after DiI crystals were placed in the medial septal area of the brain, fluorescent labeling was observed on fibers that originate in the VNO, extend along the nasal septum, grouped in distinct fascicles, make a sharp ventral turn across the cribriform plate, converge onto the ventromedial aspect of
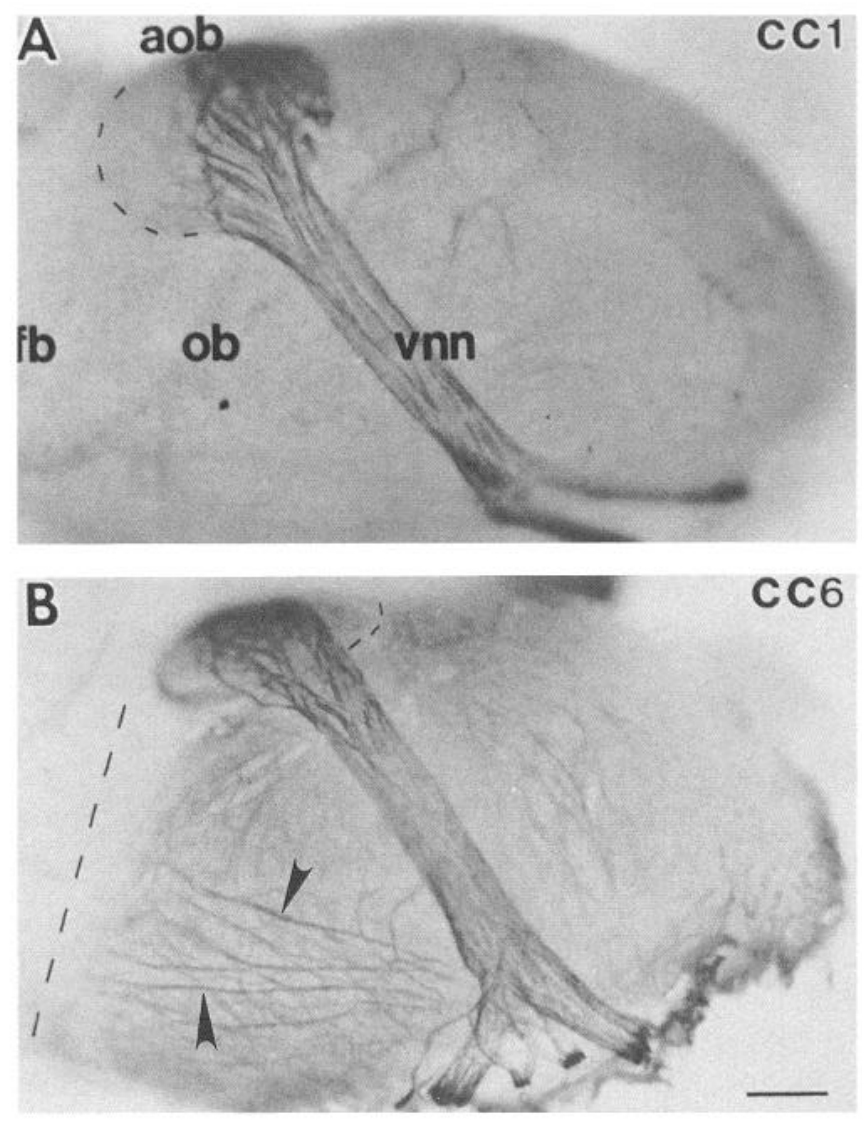

Figure 2. Whole-mount immunocytochemistry of the medial surface of the P0 rat olfactory bulbs (rostral, right; dorsal, up). $\mathrm{CC} 1$ antibodies (A) react with a subset of axons in the vomeronasal nerve (vnn) that terminate in the rostral accessory olfactory bulb $(a o b)$. The dashed line shows the border of the caudal AOB. CC6 antibodies $(B)$ react with a subset of axons in the VNN that terminate in the caudal $2 / 3$ of the AOB. The dashed line shows the rostral border of the AOB. CC6 antibodies also react with vomeronasal axons (arrowheads) that project caudally on the olfactory bulb $(o b)$ surface but do not enter the forebrain ( $f b)$ (dashed line). Rostral is to the right. Scale bar, $200 \mu \mathrm{m}$.

the bulb and proceed along the medial rostral forebrain (Fig. $1 D$ ). Taken together, these studies revealed an exuberant caudal projection of the embryonic vomeronasal nerve that extended far into the rostral forebrain. Postnatally, the majority of these caudal axons retracted and the projection consisted of only a few fibers.

\section{Rostrocaudal compartmentation of the accessory olfactory system}

We have previously shown that the accessory olfactory bulb can be divided into rostral and caudal compartments using $\mathrm{CC1}$ and CC6 monoclonal antibodies (Schwarting et al., 1994). Whole mount immunocytochemical analysis of $\mathrm{P} 0$ rat olfactory bulbs revealed that $\mathrm{CC} 1$ antibodies reacted with VNO axons terminating only in the rostral AOB (Fig. 2A). The caudal branch of the VNN was not reactive with $\mathrm{CC} 1$ antibodies. In contrast, CC6 antibodies reacted with $\mathrm{VNO}$ axons terminating in the central and caudal AOB, as well as with axons projecting caudally across the $\mathrm{OB}$ toward the forebrain. However, $\mathrm{CC}^{+}$axons did not cross the border between the $\mathrm{OB}$ and forebrain (Fig. 2B).

To further characterize the unique extension of the VNN visualized with DiI, we performed whole mount immunohisto- 

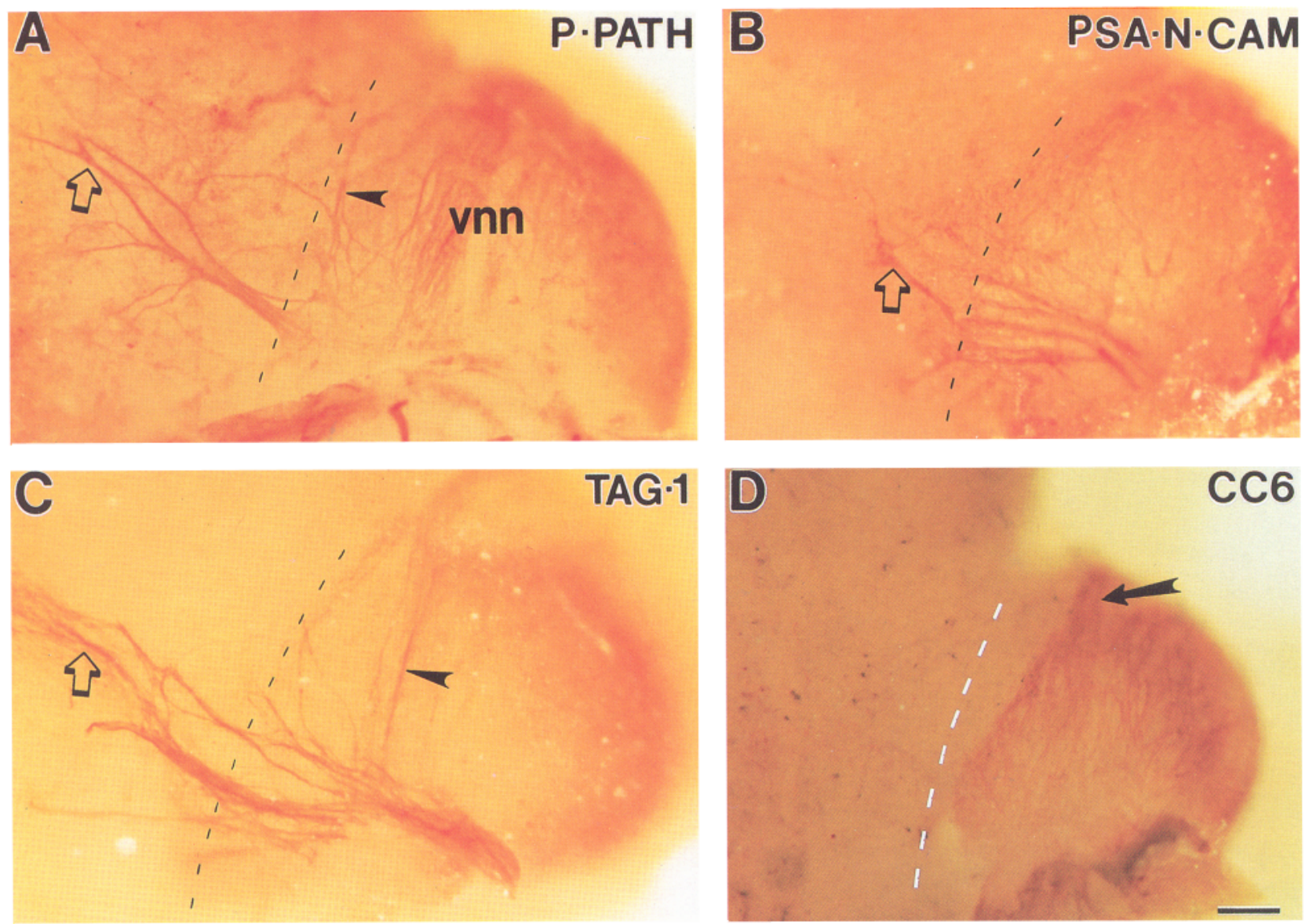

Figure 3. Whole-mount immunocytochemistry showing the medial surface of E16 rat olfactory bulbs (rostral, right; dorsal, up). P-Path antibodies $(A)$ react with all vomeronasal fibers including the main dorsal projecting vomeronasal nerve ( $v n n)$, the caudal branches that extend into the forebrain (open arrow), and other dispersed fibers that do not cross the bulb/brain border (arrowhead). PSA-N-CAM (B) is expressed only on fibers projecting into the forebrain (open arrow). TAG-1 $(C)$ is expressed on fibers that extend far into the forebrain (open arrow) and on a few fibers projecting dorsally toward the AOB (arrowhead). CC6 $(D)$ antibodies react with many fibers covering the medial surface of the OB, presumably from transient $\mathrm{CC}^{+}$OE neurons, and the main VNN (arrow). The caudal branch of the VNN is not reactive with CC6 at this age. Rostral is to the right. Scale bar in $D, 100 \mu \mathrm{m}$ for $B-D$ and $80 \mu$ for $A$.

chemistry experiments on olfactory bulb and rostral forebrain tissue from E15 to P4. At E16, P-path, PSA-N-CAM, and TAG-1 antibodies reacted with fibers that arise from the vomeronasal nerve on the medial side of the olfactory bulb, cross the brain/bulb border and continue caudally into the rostral forebrain (Fig. 3). P-Path antibodies revealed 9-0-acetylated ganglioside expression at E16, along the main vomeronasal nerve, on some areas of the bulb (indicating its presence on OE fibers), as well as on a large set of fibers that project into the rostral forebrain (Fig. 3A). P-Path antibodies also reacted with dispersed fibers between the main VNN and caudal branch of the VNN, similar to fibers in this area seen with DiI labeling (Fig. 1B). PSA-NCAM expression was localized mostly on fibers that extend from the ventromedial portion of the bulb and continue in a caudal direction towards the medial septal area of the brain (Fig. $3 B$ ). There was some PSA-N-CAM reactivity on OE axons projecting to the main olfactory bulb, although none was detectable in the main vomeronasal nerve. TAG-1 was expressed along the fibers that arise from the nasal cavity and project to the rostral forebrain in a caudal trajectory (Fig. $3 C$ ). TAG-1 staining was also visible on a few fibers within the main vomeronasal nerve that extended dorsally towards the AOB. In addition, both the mitral cell layer of the bulb as well as the lateral olfactory tract (on lateral side-not shown) expressed TAG-1. At E16, CC6 antibodies reacted with many olfactory axons (Fig. $3 D$ ) as shown by the abundance of dispersed fibers along the rostral surface of the $\mathrm{OB}$. $\mathrm{A} \mathrm{CC}^{+}$vomeronasal nerve bundle was visible growing dorsally to the AOB; however, the caudal branch of the VNN did not react with CC6 antibodies at this age.

\section{LHRH neurons migrate on the caudal branch of the VNN}

Double-label whole mount immunocytochemical studies were carried out to determine whether the route taken by migrating LHRH neurons was related to the caudal branch of the VNN. At E18, many LHRH neurons (Fig. 4A) were observed along the medial $\mathrm{OB}$ and forebrain. P-Path positive fibers were seen spreading dorsally on the caudal half of the bulb (Fig. $4 B$ ). The fibers converged at the brain/bulb border before projecting caudally into the rostral forebrain. Double labeling with LR1 antibody illustrated a clear association of LHRH neurons with these caudally directed fibers on the surface of the OB, but most LHRH neurons at this age had migrated into the forebrain beyond the terminals of P-path ${ }^{+}$axons. PSA-N-CAM expression was also visible on the caudal branches of the VNN at E18 (Fig. $4 C$ ), although no expression is visible on the main VNN. By E18, (Fig. 4D) the TAG-1 positive fibers were still visible cross- 

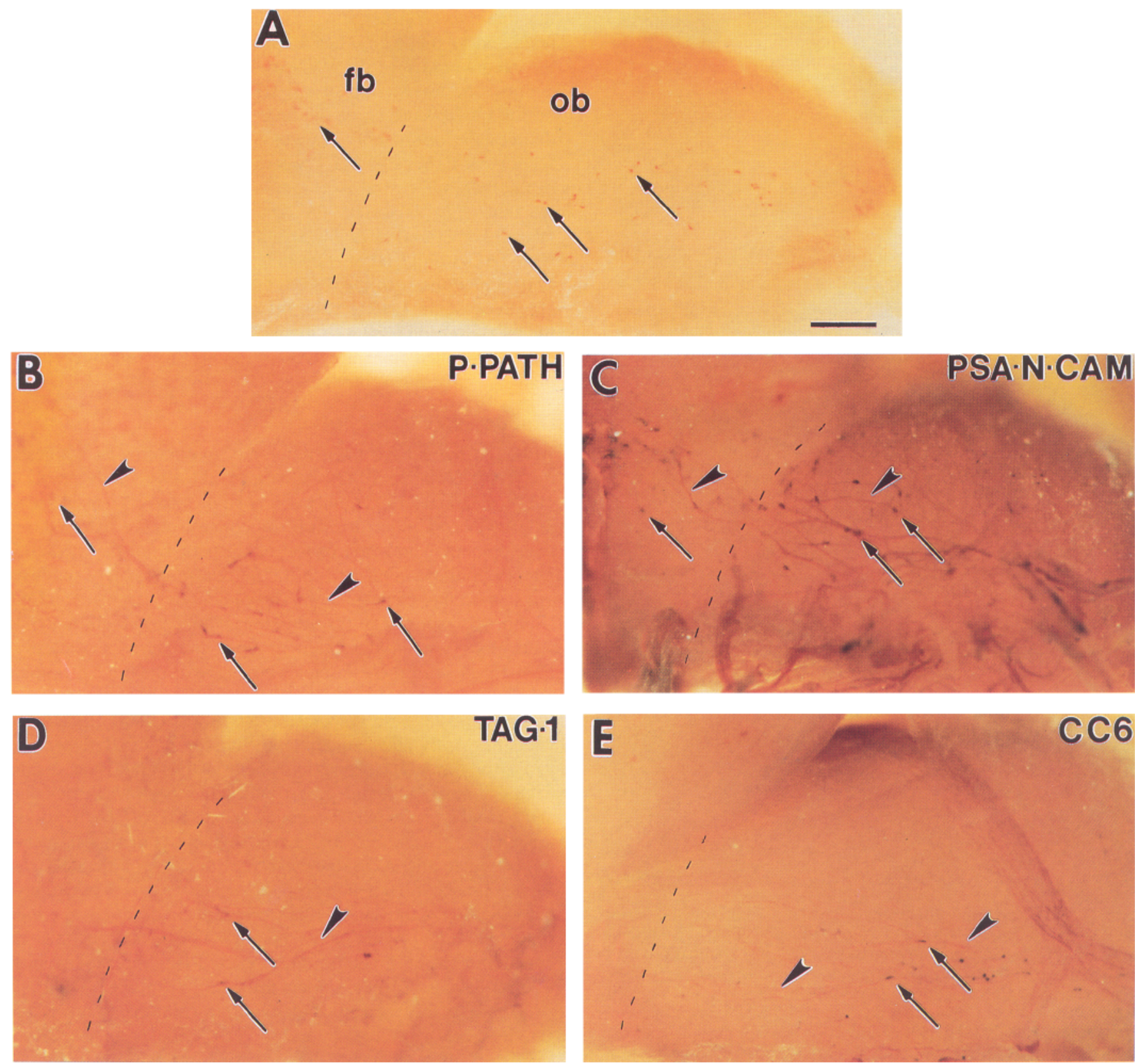

Figure 4. Double-label immunocytochemistry of the medial surface of E18 olfactory bulbs (rostral, right; dorsal, up). A, Many LHRH neurons (arrows) are evident on the medial surface of the olfactory bulb $(o b)$ and in the forebrain $(f b)$ where they begin to migrate laterally into the septum. (The forebrain LHRH cells are not as distinct because many are up to $100 \mu \mathrm{m}$ below the surface). At this age, P-path $(B)$ antibodies react with many caudal vomeronasal fibers (arrowheads). LHRH neurons appear to be in contact with these caudal fibers as they cross the OB and enter the forebrain, but at this age many neurons have migrated beyond the caudal-most extension of the P-path axons. LHRH neurons also appear to be in contact with PSA-N-CAM ${ }^{+}(C)$, TAG- $1^{+}(D)$, and $\mathrm{CC}^{+}(E)$ caudal branches of the VNN. Rostral is to the right. Note that TAG-1 expression is considerably diminished at this age and that $\mathrm{CC}^{+}$axons do not project into the forebrain. Scale bar, $200 \mu \mathrm{m}$.

ing the bulb and rostral forebrain, although the intensity of the staining diminished significantly from E16. The TAG-1 expression on the main vomeronasal nerve and AOB seen at E16 had disappeared completely. At E18, several CC6 ${ }^{+}$fibers (Fig. 4E) branched from the main VNN and continued caudally until they reached the brain/bulb border where CC6 expression abruptly ended. Double-labeled whole mount immunohistochemistry studies showed that LHRH neurons were also in contact with the $\mathrm{CC6}^{+}$caudal branches of the VNN. These studies demonstrate that LHRH neurons are consistently in contact with the caudal branches of the VNN, regardless of which antibody is used to visualize them.

\section{Postnatal retraction of the caudal VNN}

At P4, P-path antibodies reacted with all elements of the VNO projection. 9-0-Acetyl gangliosides were expressed on the main VNN, on many caudal fibers that do not extend into the forebrain, and on one or two axons that project into the forebrain (Fig. 5A). The fibers that failed to grow into the forebrain look similar to the $\mathrm{CC6}^{+}$axons at P0 that also terminated in the 

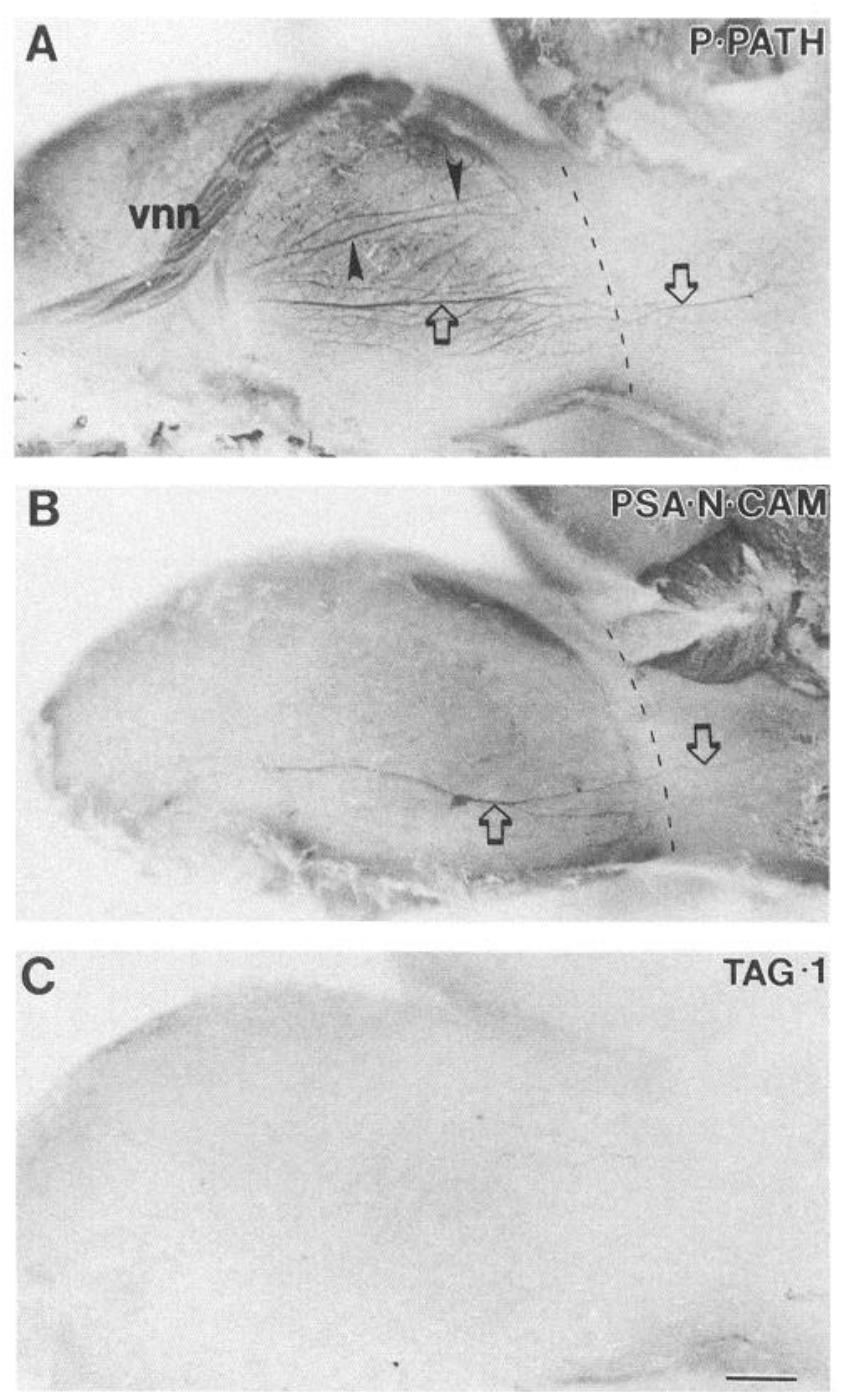

Figure 5. Whole-mount immunocytochemistry of the medial surface of the P4 rat olfactory bulb $(o b)$ (rostrăl, left; dorsal, up). P-Path antibodies react with the main vomeronasal nerve (vnn) and two groups of the caudal VNN fibers $(A)$. Some caudal fibers (arrowheads) fail to extend into the forebrain (identically to $\mathrm{CC}^{+}$fibers at P0; Fig. $2 B$ ). Other caudal branches (open arrows) react with VNN fibers that enter the forebrain. PSA-N-CAM $(B)$ is only expressed on the subset of caudal fibers that project into the forebrain (open arrows). These axons no longer express TAG-1 $(C)$. Although double-label studies still reveal occasional LHRH neurons on the medial olfactory bulb surface at this age, migration of LHRH neurons is essentially complete. The dashed line shows the border of the olfactory bulb and forebrain. Rostral is to the left. Scale bar, $400 \mu \mathrm{m}$.

caudal OB. PSA-N-CAM staining diminished at P4 (Fig. 5B). The main vomeronasal nerve was still negative while a few fibers extended across the bulb and continued into the rostral forebrain. These fibers looked identical to the subset of P-path ${ }^{+}$fibers that projected into the medial forebrain at this age. TAG-1 expression on caudal branches of the VNN had completely disappeared at P4 (Fig. 5C).

\section{Association of LHRH neurons with TAG-1 positive fibers}

Of the 4 axon markers used here, PSA-N-CAM and TAG- 1 were expressed on the smallest subset of vomeronasal axons. They were almost exclusively confined to the axons that projected into the forebrain. Furthermore, TAG-1 expression was temporally restricted to the time when LHRH neurons were migrating on the caudal branch of the VNN in the rat (Tobet et al., 1992). To confirm the association of LHRH neurons and TAG- $1^{+}$axons on the medial surface of the bulb and forebrain, we performed double-label immunofluorescence experiments on horizontal sections of E16 rats. TAG-1 was expressed on the medial surface of the bulb and rostral forebrain as well as in the mitral cells in the bulb (Fig. 6A). The TAG-1 ${ }^{+}$medial fiber track dispersed laterally as it ended in the lamina terminalis. Double-labeling with TAG-1 and LHRH (Fig. 6B) showed a close association of LHRH neurons with the TAG- $1^{+}$fibers in the rostral forebrain. However, some LHRH cells may be associated with TAG-1 ${ }^{-}$ fibers. Before migrating to the septal/preoptic area, LHRH neurons dissociated from the TAG- ${ }^{+}$axons and continued to migrate laterally and ventrally.

\section{Discussion}

Although there have been numerous morphological studies of the VNO (Adams, 1992; Garrosa et al., 1992; Salazar et al., 1992; Mendoza, 1993), only a few studies have focused on the outgrowth and trajectories of vomeronasal nerve axons during development. The vomeronasal organ buds from the medial olfactory epithelium on E13 in the rat and immediately sends axons toward the telencephalon. Throughout development and into adulthood, axons from the vomeronasal neuroepithelium can be seen along the nasal septum grouped into large fascicles. At E14-15, most of these fascicles converge at the cribriform plate and project across the medial surface of the olfactory bulb, in a fan-like array. These dispersed VNN fibers extend dorsally toward the AOB and caudally into the forebrain. Between E15 and E16, the VNO projection begins to separate into distinct axonal groups; a dorsal group which has begun to fasciculate and a caudal group which remains dispersed. At E16, the dorsal branch has fasciculated into a recognizable vomeronasal nerve, projecting to the AOB on the dorsal surface of the $O B$. The caudal branch of the vomeronasal nerve has not fasciculated, but extends far into the medial forebrain. In addition, there are some individual VNN fibers on the OB surface between the dorsal and caudal branches. Previous DiI labeling studies in the E16 rat has also revealed fibers projecting from the VNO to the $\mathrm{AOB}$ and to the ventromedial forebrain (Marchand and Belanger, 1991).

Figure 7 is a schematic diagram depicting the four subsets of vomeronasal axons. The dorsal branch of the VNN is shown terminating in two distinct $\mathrm{AOB}$ compartments: $\mathrm{CC}^{+}$axons terminate only in the rostral $\mathrm{AOB}$ and $\mathrm{CC}^{+}$axons terminate preferentially in the caudal AOB. The caudal branch of the VNN is also divided into two groups; $\mathrm{CC}^{+}$axons terminate in the olfactory bulb, whereas TAG- $1^{+}$, PSA-N-CAM ${ }^{+}$axons extend into the basal forebrain. LHRH neurons are shown migrating in the nasal cavity on the large fascicles of the VNN. After crossing the cribriform plate, LHRH neurons migrate on the subset of caudal axons that express TAG-1 and PSA-N-CAM. Doublelabel studies have not been performed (each of these antibodies is a mouse IgM) but it is likely that TAG-1 and PSA-N-CAM are expressed on the same caudal-oriented fibers. CC6 is expressed on a different group of caudal fibers, although some $\mathrm{CC}^{+}$fibers may fasciculate with TAG- ${ }^{+}$, PSA-N-CAM ${ }^{+}$fibers as shown in Figure 7. Thus, LHRH neurons are seen in contact with axons that express all three of these epitopes. Alternatively, it is possible that $\mathrm{CC} 6$ glycoconjugates are coexpressed on the 

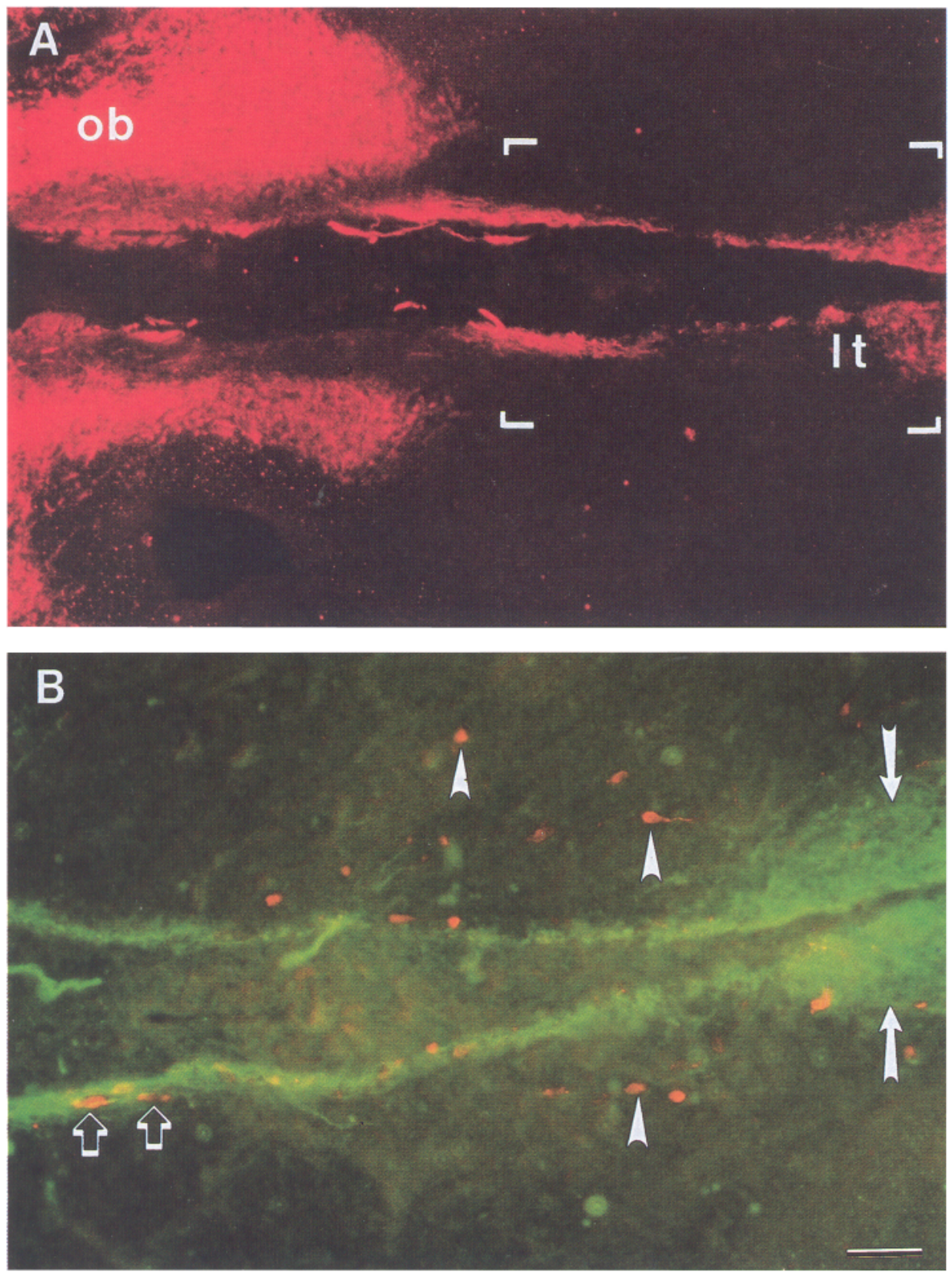

Figure 6. Double-label immunofluorescence of TAG-1 axons and LHRH neurons at E16. In a horizontal section of the olfactory bulb $(o b)$ and forebrain $(A)$, TAG-1 is expressed on fibers that extend along the medial surface of the $\mathrm{OB}$ and forebrain, terminating in the lamina terminalis $(l t)$. TAG-1 is also expressed on mitral/tufted cells in the $\mathrm{OB}$ and the lateral olfactory tract. $(B)$ In an adjacent section of an area similar to the box in A, LHRH neurons are seen in contact (open arrows) with TAG- $1^{+}$axons, others are dissociated (arrowheads) from these fibers and move laterally before they reach the TAG-1 terminal arbors (arrows). Rostral is to the left. Scale bar: $100 \mu \mathrm{m}$ in $A, 50 \mu \mathrm{m}$ in $B$. proximal segment of $\mathrm{TAG}-1^{+}$, PSA-N-CAM ${ }^{+}$axons. This pattern is similar to the restriction of TAG-1 expression to the ipsilateral portion of commissural axons in the developing spinal cord (Dodd et al., 1988). A second question that remains unresolved is whether the caudal fibers represent collateral branches of axons projecting to the AOB, or axons from a subset of sensory neurons that project directly from the VNO exclusively into the basal forebrain.

The four epitopes shown in Figure 7 are expressed on particular subsets of vomeronasal axons; in contrast, P-path is expressed on some olfactory epithelial axons and both of the caudal and dorsal branches of the VNN throughout the period of LHRH neuron migration. Furthermore, CC6 glycoconjugates are not expressed on the caudal branch of the VNN before E18, when most of the migration takes place. Taken together, these data suggest that neither P-path nor CC6 glycoconjugates play a direct role in LHRH neuron migration.
The TAG- ${ }^{+} /$PSA-N-CAM ${ }^{+}$subset represents a small fraction of the total vomeronasal projection, but virtually all of the axonal guides for LHRH neuron migration into the basal forebrain. Although TAG-1 is expressed on a few neurons in the OE and on mitral cells in the OB, within the VNO projection, it is preferentially expressed spatially and temporally along the route of LHRH neuron migration. However, a few fibers in the main VNN also express TAG-1, suggesting that TAG-1 expression alone may not be sufficient to designate the LHRH neuron migration pathway. PSA-N-CAM immunoreactivity is seen on many cells in the $\mathrm{OE}, \mathrm{OB}$ and forebrain but is also restricted to the subset of VNO axons that project caudally. These axons rarely, if ever, react with LHRH antibodies at any age between E14 and P14 in the rat, although Zheng et al. (1988) have observed the presence of some LHRH immunoreactive axons on the medial surface of the OB in adult rats. We have not found evidence that structural elements such as glial processes or blood 


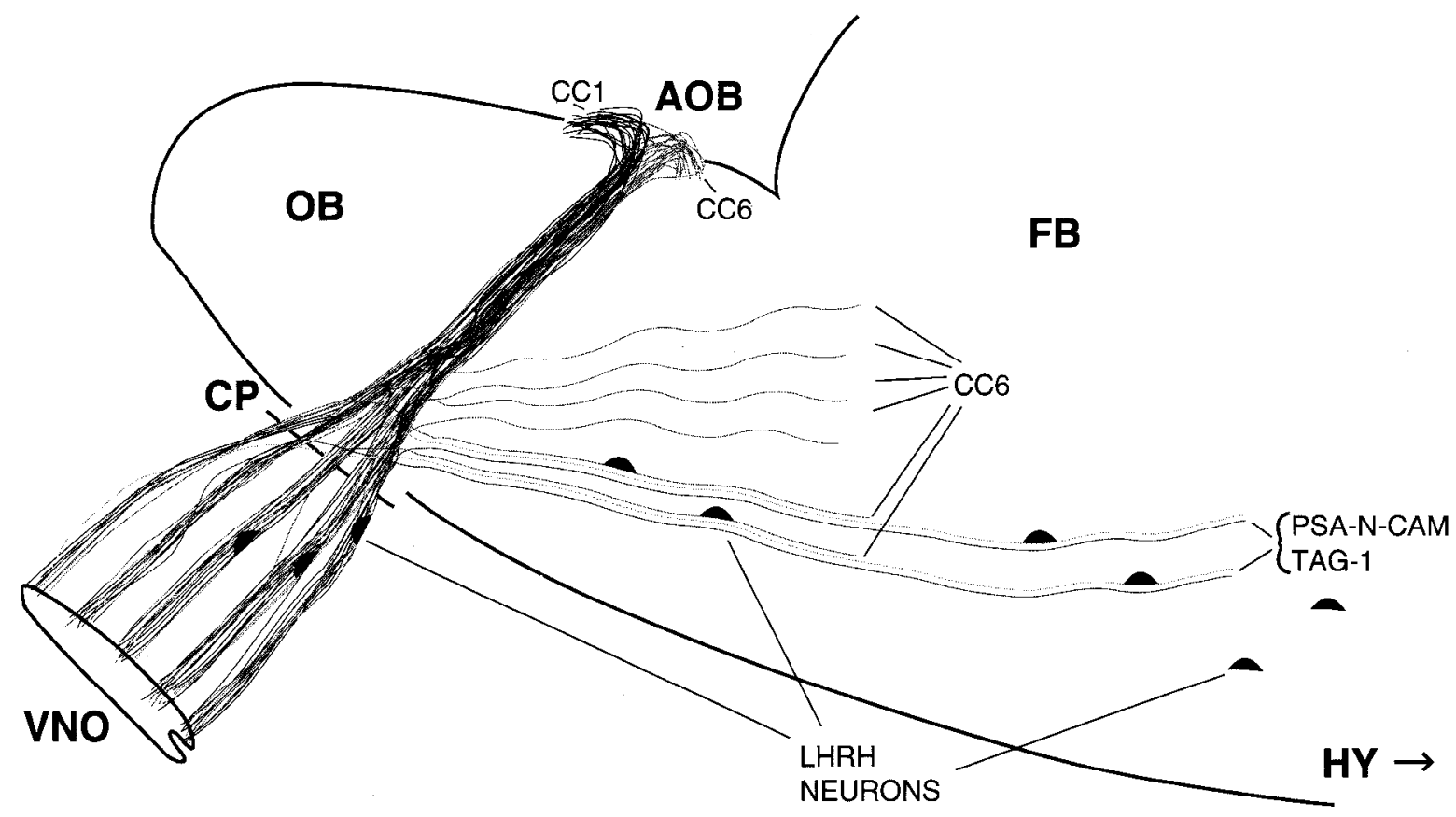

Figure 7. Schematic diagram of the four groups of vomeronasal axons, including the proposed pathway of LHRH neuron migration from the vomeronasal organ $(V N O)$ towards the hypothalamus $(H Y)$. Two groups extend to the accessory olfactory bulb $(A O B)$ : $C C 1^{+}$fibers terminate in the rostral portion of the AOB; $\mathrm{CC}^{+}$fibers project to the caudal AOB. The caudal branch of the vomeronasal nerve divides into one set of $\mathrm{CC}^{+}$ fibers which stops at the olfactory bulb $(O B)$ /forebrain $(F B)$ border and another set of PSA-N-CAM ${ }^{+} / \mathrm{TAG}-1^{+}$fibers which continues into the lamina terminalis (rostral to the hypothalamus). LHRH neurons migrate utilizing the PSA-N-CAM ${ }^{+} / \mathrm{TAG}^{+}{ }^{+}$subset of vomeronasal axons. CP, Cribriform plate.

vessels are associated with this migration pathway. Although these studies have not been performed at the ultrastructural level, double-label experiments illustrate that, at the ages when most LHRH neurons migrate across the cribriform plate to the forebrain (E14-16), they are usually in close association with vomeronasal axons that express TAG-1 and PSA-N-CAM.

Several reports in the developing chick have suggested a potential role for PSA-N-CAM in LHRH neuron migration. Murakami et al. (1991) have indicated the possibility that PSA-N-C $\Lambda \mathrm{M}$ aids in the migration of LHRH cells in the main olfactory system. Because polysialated forms of N-CAM are associated with developing systems and are thought to be less adhesive, Murakami et al. suggest that PSA-N-CAM plays a direct role in LHRH neuron migration. The role for PSA-NCAM (as well as other cell adhesion molecules) is also supported by Norgren and Brackenbury (1993). They note that NCAMs may not be involved in the migration of LIIRII neurons from the nasal cavity to the cribriform plate, but are perhaps significantly involved in their movement along the medial surface of the olfactory bulb and rostral forebrain.

PSA-N-CAM has also been implicated in axon branching during innervation of chick muscle (Landmesser et al., 1990). Recent studies of motor axon sorting indicate that PSA associated with N-CAM probably plays a permissive role (Tang et al., 1994). By attenuating axon-axon interactions, PSA may facilitate the action of other guidance cues during development. In the accessory olfactory system, PSA-N-CAM expression is restricted to the less fasciculated, caudal VNN axons and may induce this group of fibers to branch off of the main VNN, allowing other adhesion mechanisms to influence their trajectories. It is likely that other factors such as soluble chemotropic molecules, extracellular matrix molecules, and adjacent neurons and glial cells, may also be involved in defasciculation and directed outgrowth of the caudal VNN.

TAG-1 is an adhesion molecule that is capable of modulating cell-cell interactions (Yamamoto et al., 1986; Dodd et al., 1988). It is attached to the cell surface by a glycosylphosphatidylinositol linkage and can be released into the extracellular space (Furley et al., 1990; Karagogeos et al., 1991). It was recently shown that dorsal root ganglion neurite outgrowth on a TAG-1 substrate requires the function of $\mathrm{L} 1$ adhesion molecules and $\beta 1$-containing integrins (Felsenfeld et al., 1994). Whether or not TAG-1 mediated interaction can also play a role in cell migration is not known. In the accessory olfactory system, $\mathrm{Ll}$ is expressed on the caudal branch of the vomeronasal nerve (data not shown), much like TAG-1 and PSA-N-CAM, but there is no indication that rat LHRH neurons express any ligands that would allow them to interact with these axons.

During development, LHRH neurons are known to migrate from the olfactory placode to the forebrain in most mammalian species (Schwanzel-Fukuda and Pfaff, 1989). Kallmann Syndrome is an X-linked genetic disorder that is characterized by the association of hypogonadotropic hypogonadism with anosmia. In one striking case, this was correlated with the failure of LHRH neurons to arrive in the forebrain. (Schwanzel-Fukuda et al., 1989). Recent studies have shown that, in addition to LHRH neurons, there are other neurons that migrate along the same track from the VNO to the forebrain (Murakami and Arai, 1994). We have recently found that one population of non-LHRH neurons which appear to migrate along vomeronasal nerves contains GABA (Tobet, Chickering, and Schwarting, unpublished observations). However, we have not yet characterized their association with $\mathrm{TAG}-1^{+}$, PSA-N-CAM ${ }^{+}$, or $\mathrm{CC}^{+}$fibers.

In summary, transient axons derived from neurons in the 
VNO project caudally across the forebrain of the embryonic rat. After migrating into the forebrain in association with the TAG$1^{+}$, PSA-N-CAM ${ }^{+}$axons, the majority of LHRH neurons continue to migrate laterally and ventrally into the preoptic area/ anterior hypothalamus. Further in vivo and in vitro experiments are presently underway to further explore the role of TAG-1, PSA-N-CAM and possible chemotropic factors in LHRH neuron migration from the nasal cavity to the forebrain in the developing rodent.

\section{References}

Adams DR (1992) Fine structure of the vomeronasal and septal olfactory epithelia and of glandular structures. Micro Res Tech 23:86-97.

Akutsu S, Takada M, Ohki-Hamazaki H, Murakami S, Arai Y (1992) Origin of luteinizing hormone-releasing hormone (LHRH) neurons in the chick embryo: effect of the olfactory ablation. Neurosci Lett 142: 241-244.

Bronner-Fraser M (1992) Environmental influences on neural crest cell migration. J Neurobiol 24:233-247.

Daikoku S, Koide I, Chikamori-Aoyama M, Shimomura Y (1993) Migration of LHRH neurons derived from the olfactory placode in rats. Arch Histol Cytol 56:353-370.

Dodd J, Morton SB, Karagogeos D, Yamamoto M; Jessell TM (1988) Spatial regulation of axonal glycoprotein expression on subsets of embryonic spinal neurons. Neuron 1:105-116.

Felsenfeld DP, Hynes MA, Skoler KM, Furley AJ, Jessell TM (1994) TAG-1 can mediate homophilic binding, but neurite outgrowth on TAG-1 requires an $L 1$-like molecule and $\beta 1$ integrins. Neuron 12: 674-690.

Furley AJ, Morton SB, Manalo D, Karagogeos D, Dodd J, Jessell TM (1990) The axonal glycoprotein TAG-1 is an immunoglobulin superfamily member with neurite outgrowth-promoting activity. Cell $61: 157-170$.

Garrosa M, Iniguez C, Fernandez JM, Gayoso MJ (1992) Developmental stages of the vomeronasal organ in the rat: a light and electron microscopic study. J Hirnforn 33:123-132.

Gong Q, Shipley MT (1995) Evidence that pioneer olfactory axons regulate telencephalon cell cycle kinetics to induce the formation of olfactory bulb. Neuron 14:91-101.

Halpern M (1987) The organization and function of the vomeronasal system. Rev Neurosci 10:325-362.

Hatten ME (1993) The role of migration in central nervous system neuronal development. Curr Opinion Neurobiol 3:38-44.

Karagogeos D, Morton SB, Casano F, Dodd J, Jessell TM (1991) Developmental expression of the axonal glycoprotein TAG-1: differential regulation by central and peripheral neurons in vitro. Development 112:51-67

Landmesser L, Dahm L, Tang J, Rutishauser U (1990) Polysialic acid as a regulator of intramuscular nerve branching during embryonic development. Neuron 4:655-667.

Marchand R, Belanger MC (1991) Ontogenesis of the axonal circuitry associated with the olfactory system of the rat embryo. Neurosci Lett 129:285-290.

Mendoza AS (1993) Morphological studies on the rodent main and accessory olfactory systems: the regio olfactoria and vomeronasal organ. Ann Anat 175:425-446.

Meredith M (1986) Vomeronasal organ removal before sexual experience impairs male hamster mating behavior. Physiol Behav 36:737743.

Murakami S, Arai Y (1994) Direct cridence for the migration of LHRH neurons from the nasal region to the forebrain in the chick embryo: a carbocyanine dye analysis. Neurosci Res 19:331-338.

Murakami S, Seki T, Wakabayashi K, Arai Y (1991) The ontogeny of luteinizing hormone-releasing hormone (LHRH) producing neurons in the chick embryo: possible evidence for migrating LHRH neurons from the olfactory epithelium expressing a highly polysialylated neural cell adhesion molecule. Neurosci Res 12:421-431.

Norgren RB, Brackenbury R (1993) Cell adhesion molecules and the migration of LHRH neurons during development. Dev Biol 160:377387.
Norgren RB, Gao C (1994) LHRH neuronal subtypes have multiple origins in chickens. Dev Biol 165:735-738.

Norgren RB, Lehman MN (1991) Neurons that migrate from the olfactory epitheliun in the chick express luteinizing hormone-releasing hormone. Endocrinology 128:1676-1678.

Rakic P (1990) Principles of neural cell migration. Experientia 46:882891.

Salazar I, Barber PC, Cifuentes JM (1992) Anatomical and immunohistological demonstration of the primary neural connections of the vomeronasal organ in the dog. Anat Rec 233:309-313.

Santacana M, Heredia M, Valverde F (1992) Transient pattern of exuberant projections of olfactory axons during development in the rat. Dev Brain Res 70:213-222.

Schwanzel-Fukuda M, Pfaff DW (1989) Origin of luteinizing hormonereleasing hormone neurons. Nature 338:161-164.

Schwanzel-Fukuda M, Bick D, Pfaff DW (1989) Luteinizing hormonereleasing hormone (LHRH)-expressing cells do not migrate normally in an inherited hypogonadal (Kallmann) syndrome. Mol Brain Res 6:311-326.

Schwanzel-Fukuda M, Abraham S, Crossin KL, Edelman GM, Pfaff DW (1992) Immunocytochemical demonstration of neural cell adhesion molecule (NCAM) along the migration route of luteinizing hormone-releasing hormone (LHRH) neurons in mice. J Comp Neurol 321:1-18.

Schwanzel-Fukuda M, Reinhard GR, Abraham S, Crossin KL, Edelman GM, Pfaff DW (1994) Antibody to neural cell adhesion molecule can disrupt the migration of luteinizing hormone-releasing hormone neurons into the mouse brain. J Comp Neurol 342:174-185.

Schwarting GA, Deutsch G, Gattey DM, Crandall JE (1992) Glycoconjugates are stage- and position-specific cell surface molecules in the developing olfactory system, 2 : unique carbohydrate antigens are topographic markers for selective projection patterns of olfactory axons. J Neurobiol 23:130-142.

Schwarting GA, Drinkwater D, Crandall JE (1994) A unique neuronal glycolipid defines rostrocaudal compartmentalization in the accessory olfactory system of rats. Dev Brain Res 78:191-200.

Tang J, Rutishauser U, Landmesser L (1994) Polysialic acid regulates growth cone behavior during sorting of motor axons in the plexus region. Neuron 13:405-414.

Tobet SA, Crandall JE, Schwarting GA (1992) Relationship of migrating luteinizing hormone-releasing hormone neurons to unique olfactory system glycoconjugates in embryonic rats. Dev Biol 155: $471-482$.

Wirsig CR, Getchell TB (1986) Amphibian terminal nerve: distribution revealed by LHRH an AChE markers. Brain Res 385:10-21.

Wirsig-Wichmann CR (1993) Nervus terminalis lesions. I. No effect on pheromonally induced testosterone surges in the male hamster. Physiol Behav 53:251-255.

Wray S, Grant P, Gainer H (1989) Evidence that cells expressing luteinizing hormone-releasing hormone mRNA in the mouse are derived from progenitor cells in the olfactory placode. Proc Natl Acad Sci USA 86:8132-8136.

Wray S, Key S, Qualls R, Fueshko SM (1994) A subset of peripherin positive olfactory axons delineates the luteinizing hormone releasing hormone neuronal migratory pathway in developing mouse. Dev Biol $166: 349-354$

Wysocki CI, Meredith M (1986) The vomeronasal system. In: Neurobiology of taste and smell (Finger TE, Silver WL, eds), pp 125150. New York: Wiley.

Yamamoto M, Boyer A, Schwarting GA (1985) Fucose-containing glycolipids are stage- and region-specific antigens in developing embryonic brain of rodents. Proc Natl Acad Sci USA 82:3045-3049.

Yamamoto M, Boyer AM, Crandall JE, Edwards M, Tanaka II (1986) Distribution of stage specific neurite-associated proteins in the developing murine nervous system recognized by a monoclonal antibody. J Neurosci 6:3576-3594.

Yamamoto M, Schwarting GA, Crandall JE (1994) Altered 9-O acetylation of disialogangliosides in cerebellar Purkinje cells of the nervous mutant mouse. Brain Res 662:223 232.

Zheng LM, Caldani M, Jourdan F (1988) Immunocytochemical identification of luteinizing hormone-releasing hormone-positive fibres and terminals in the olfactory system of the rat. Neuroscience 24: $567-578$. 